\title{
The CFVib Experiment: Control of Fluids in Microgravity with Vibrations
}

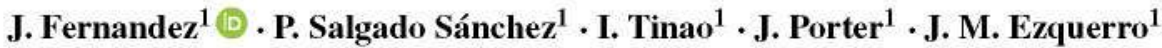

\begin{abstract}
The Control of Fluids in Microgravity with Vibrations (CFVib) experiment was selected for the 2016 Fly Your Thesis! programme as part of the 65th ESA Parabolic Flight Campaign. The aim of the project is to observe the potentially complex behaviour of vibrated liquids in weightless environments and to investigate the extent to which smallamplitude vibrations can be used to influence and control this behaviour. Piezoelectric materials are used to generate high-frequency vibrations to drive surface waves and largescale reorientation of the interface. The theory of vibroequilibria, which treats the quasi-stationary surface configurations achieved by this reorientation, was used to predict interesting parameter regimes and interpret fluid behaviour. Here we describe the scientific motivation, objectives, and design of the experiment.
\end{abstract}

Keywords Fluid mechanics - Microgravity experiment . Instability · Vibroequilibria · Parabolic flight

\section{Introduction}

By its nature fluid moves easily, adjusting its shape and position in response to external forces. On earth, the most prominent large-scale external force is gravity and fluid configurations generally evolve so as to minimize their gravitational potential energy. The effects of this are familiar to everyone. An undisturbed body of liquid, for example, whether part of the natural landscape or held in a container, will minimize its height by pooling in the lowest configuration possible, maintaining a (nearly) flat horizontal interface with the surrounding air (or other lighter fluid). Although this picture can be complicated by surface tension, which is important on small lengthscales, and various other physical processes like buoyancy, diffusion and evaporation, the basic flat interface selected at leading order in common situations is remarkably simple.

Periodic vibrations represent an interesting class of external forcing that can perturb or disrupt this initial (static) surface configuration. Vibrations have the potential to both induce instability (Faraday 1831; Miles and Henderson 1990; Vega et al. 2001) and suppress it (Gresho and Sani 1970; Wolf 1969; 1970), and to affect important physical processes like mixing and mass transfer. In the laboratory, periodic vibrations can be introduced in a deliberate and controlled way and the effects of amplitude, phase, and frequency content systematically investigated.

The effects of vibration on a mass of fluid can be roughly divided according to the frequency and the orientation of the forcing. Low-frequency vibrations (provided they are not purely orthogonal to the surface) tend to excite backand-forth motion along the axis of the vibration, or sloshing modes (Miles 1984; Funakoshi and Inoue 1988; Feng 1997). Higher frequencies excite surface (or interfacial) waves of shorter wavelength, which may be either harmonic or subharmonic (Barnard and Pritchard 1972; Miles and Henderson 1990). The smaller lengthscale of these waves leads to the possibility of pattern selection and associated dynamics, which has been a subject of much interest (Edwards and Fauve 1994; Kudrolli et al. 1998; Arbell and Fineberg 2002). 
The orientation of the forcing is a key factor in the response of the fluid. If forcing is applied perpendicular to a large flat surface then its initial effect is homogeneous and isotropic. The flat surface will persist, but undergoes a parametric instability (Benjamin and Ursell 1954) at a certain critical forcing amplitude to (typically) subharmonic surfaces waves, which appear with uniform amplitude across the surface. Horizontal (more generally, non-vertical) forcing has more complex effects, producing harmonic (synchronous) waves and, in many situations, subharmonic waves such as cross-waves (Garrett 1970; Jones 1984; Porter et al. 2012). In contrast to the case of vertical (perpendicular) motion, horizontal forcing typically produces nonuniform wave fields concentrated near the moving boundaries or wavemakers. An interesting exception to this is the appearance at a critical forcing amplitude of a Kelvin-Helmholtz type instability in heterogeneous systems, such as a stably stratified layer of immiscible fluids, that initially produces quasi-stationary frozen waves (Wolf 1969; Lyubimov and Cherepanov 1986; Talib et al. 2007; Gaponenko et al. 2015; Gaponenko and Shevtsova 2016). Sufficiently far from the boundaries these frozen waves are of uniform amplitude.

In addition to surface waves, which oscillate on the fast timescale set by the forcing, periodic vibrations can drive a slower reorientation of the fluid toward a new quasi-steady equilibrium distinct from the hydrostatic one. This phenomenon, observed by Faraday (1831) in the flattening of fluid drops suspended beneath a vibrating plate, is known as the vibroequilibria effect. Like frozen waves, vibroequilibria are supported by an oscillatory velocity field generated by the applied vibrations. It is the spatial inhomogeneity of this velocity field, and the corresponding oscillatory pressure gradient, that leads to deformation of the surface. Recent analysis (Fernández et al. 2017), using both numerical and theoretical techniques, found that, depending on fluid volume, vibroequilibria in horizontally vibrated rectangular containers can suffer a saddle-node instability that initiates a disordered transition to one of many possible asymmetric states. In low-viscosity fluids, surface wave dynamics can also promote the destruction of vibroequilibria states, with a strong correlation found between modulated subharmonic surfaces waves and the (potentially unbounded) growth of the first odd sloshing mode.

In microgravity environments, fluid behaves quite differently than it does on earth. Surface tension effects are no longer suppressed by gravity and there is no preference for flat surfaces (interfaces). A free-floating mass of liquid will, instead, minimize its surface energy by taking a spherical shape, while contact forces may cause a confined liquid to fully cover (wet) the solid boundaries. The distinction between vertical and horizontal forcing is of minimal use since most surfaces will be curved and, thus, the forcing mechanisms driving surface instabilities will generally be inhomogeneous. Among other things, this means that the distinction between Faraday waves, which are associated with uniform vertical forcing, and cross-waves, which arise in horizontally forced systems, is blurred. Other phenomena, like frozen waves, are altered as well, as shown by recent experiments (Gandikota et al. 2014) in near-critical hydrogen that found the frozen wave amplitude increases with decreasing gravity level, eventually approaching the lengthscale of the container (domain) in microgravity conditions.

The absence of a constant gravitational force has a marked effect on the vibroequilibria phenomenon, which is no longer suppressed by the high gravitational cost of changing surface shape (height). In microgravity, vibrational energy primarily competes with surface and contact energy, and these may be comparable in magnitude for small to moderate forcing amplitudes. The ability of fluid masses to significantly adjust their shape in response to vibrations, combined with the selection of curved hydrostatic surfaces due to surface tension, means that fluid management in microgravity is considerably more complicated than it is on earth.

Although vibrations are frequently unwanted in a microgravity environment, they may also be put to good use. They are under consideration to help reduce muscle and bone loss in astronauts, for example, and can mimic the effects of gravity in other ways too (Beysens 2006), inducing convection patterns similar to Rayleigh-Bénard (buoyancy driven) convection in a fluid confined between two plates of different temperature, for instance. Vibrations generate mean flows that affect mixing, heat transfer, and phase separation, among other important physical processes. Further understanding of the various effects of vibrations on fluids may make it possible to use these effects for advantage in microgravity - not only to avoid undesired phenomena, but to deliberately manipulate and manage fluids, a critical problem in space travel. The vibroequilibria effect, in particular, suggests artificial gravity since, as forcing amplitude increases, the interface typically evolves toward a more perpendicular alignment with respect to the axis of vibration, thereby restoring a preference for flat interfaces.

The CFVib experiment examines the dynamic behaviour of representative liquid-gas and liquid-liquid combinations confined in moderately small transparent acrylic containers (several centimeters across) and subjected to periodic vibrations. Relatively high frequencies (from about $50 \mathrm{~Hz}$ to $12 \mathrm{kHz}$ ) are used to drive the reorientation of the fluid interface and explore the potential for controlling it. The use of piezoelectric materials to transmit vibrations to the fluid requires less space and power than an electromagnetic shaker, making it more conducive to potential microgravity applications. On the other hand, there are challenges 
for theory and simulations since the excited motion within the container is complicated, nonuniform, and sensitive to resonance conditions.

The period of weightlessness (more than $20 \mathrm{~s}$ ) offered by a parabolic flight (Pletser et al. 2016) is well-suited to the scientific requirements of the experiment. Furthermore, the g-level cycles resulting from repeated parabolas can be treated as a natural and variable resetting of the liquid, in keeping with the idea of investigating a robust method for manipulating fluids in microgravity.

In this paper we first present an overview of the most relevant microgravity experiments and theory related to the vibroequilibria effect in microgravity. Then, the scientific objectives and experiment requirements are described, and the experiment design is presented including the containershaker assembly, data acquisition system, and electrical subsystems, as well as the operational concept and experimental procedure. A final review of the CFVib experiment design precedes a selection of preliminary results, followed by final conclusions.

\section{Prior Results and Scientific Motivation}

There have been relatively few experimental investigations of vibrated fluids in microgravity and none, that we are aware of, which look directly at the vibroequilibria effect or which used piezoelectric actuators to deliver the vibrations.

The most direct antecedent of the CFVib experiment is arguably the parabolic flight experiment of Ganiev et al. (1977), which investigated the dynamic behaviour of water, oil, and air mixtures in partially filled vibrating cylindrical cavities. The interface was studied using both lowfrequency and high-frequency vibrations applied with an electrodynamic shaker. Relatively disordered behaviour, involving multiple modes and oscillating bubbles, was observed at low frequencies $(8 \mathrm{~Hz}, 12 \mathrm{~Hz})$ while highfrequency vibrations $(600-800 \mathrm{~Hz})$ led to the formation of deep funnels or craters, which penetrated nearly to the bottom of the container. This phenomenon, which would now be recognized as a type of vibroequilibrium, was strongly resonant in character.

Experiments using the ALICE-2 instrument on the MIR space station (Zyuzgin et al. 2001; Garrabos et al. 2007) examined the influence of low-frequency vibrations $(0.2 \mathrm{~Hz}$, $1.6 \mathrm{~Hz})$ on heat propagation in supercritical fluids and observed a strong effect.

Parabolic flight experiments (Mialdun et al. 2008; Shevtsova et al. 2010a, b) demonstrated vibration-induced convection in nonuniformly heated fluid in reduced gravity using excitation frequencies of $1-12 \mathrm{~Hz}$. An extension of these efforts, the Influence of Vibrations on Diffusion in
Liquids (IVIDIL) experiments (Shevtsova 2010; Shevtsova et al. 2011, 2015) on the International Space Station (ISS) investigated the effects of vibration on diffusion and convection. Diffusion and thermodiffusion coefficients were measured for two binary mixtures and it was found that vibrations of constant frequency and amplitude can generate mean flows that affect both processes. Two methods of mass transfer were observed, depending on the vibrational forcing. With low forcing, the mass transport is controlled by the Soret effect. When the forcing is high, mass transfer is convective and the Soret effect is localized in the boundaries generating the heat flux. The importance of $g$-jitter for diffusion experiments was also evaluated and found to be largely negligible; only large transient events like docking or orbital corrections have a notable impact on such experiments.

The Vibrational Phenomena in Liquids (VIPIL) project focuses on vibrational phenomena in miscible and immiscible fluids, including heat and mass transfer. Associated parabolic flight experiments (Shevtsova et al. 2016) have found large-amplitude frozen waves that evolve into columnar structures stretching across the width of the container. The column interfaces, which are largely perpendicular to the axis of the forcing, can develop subharmonic surface waves (i.e., Faraday waves). This subharmonic instability occurs with constant gravity as well, although the average structure of the large-amplitude frozen waves then resembles a triangle wave.

Finally, we note the Capillary Flow Experiment (CFE) (Jenson et al. 2010; Weislogel et al. 2009), which demonstrated the importance of contact line boundary conditions in selecting the preferred static equilibrium of perturbed fluids and in the response of the fluid to manually imparted perturbations of various types.

On the theoretical side, there has been a considerable amount of work on vibrated fluids, including the phenomenon of vibroequilibria (Lyubimov et al. 1997; Beyer et al. 2001; Lyubimov et al. 2003; Beyer et al. 2004; Gavrilyuk et al. 2004). The approach to vibroequilibria theory is based on a time-averaged variational method that assumes an inviscid fluid and irrotational flow. The forcing frequency is taken to be much larger than the frequency of the primary sloshing mode, while incompressibility, if assumed, further requires that this forcing frequency is below the frequency of the first acoustic resonance. The Lagrangian (Hamiltonian) variational method then leads to an energy functional accounting for surface energy, vibrational energy (Kapitza 1951) and, if present, gravitational energy. The surface configurations associated with critical points of this functional are the vibroequilibria solutions.

The predictions of vibroequilibria theory for horizontally vibrated containers were tested against direct numerical 
simulations (Fernández et al. 2017), where, with large enough damping, very good agreement was found. Lowviscosity fluids support more prominent surfaces waves, which can destabilize the underlying vibroequilibria state through the excitation of sloshing modes. This instability is correlated with the presence of modulated (quasiperiodic) subharmonic surfaces waves, which are typical at onset (Varas and Vega 2007; Porter et al, 2013; Perez-Gracia et al. 2014; Tinao et al. 2014; Salgado Sánchez et al, 2016). Even without the complications of surface wave dynamics, an interesting dependence on depth was found for the symmetric connected vibroequilibria state, which develops a large central trough (crater) as forcing is increased and the fluid is pushed toward the vibrating endwalls. For shallow layers of fluid this central dip gradually reaches the container bottom, but for sufficiently deep layers the symmetric vibroequilibria state disappears through a saddle-node bifurcation, after which this central trough plunges rapidly toward the bottom in a more violent and unpredictable fashion. The transition to asymmetric vibroequilibria states found at still higher forcing is inherently hysteretic.

The various results summarized above highlight both the interesting fluid phenomena that can be observed with vibrations in microgravity environments and some of the challenges for fluid management in such situations. The aim of the CFVib experiment is to extend the level of scientific understanding in this area and, specifically, to explore the potential of small-amplitude vibrations to control liquid-air or immiscible liquid-liquid configurations.

\section{Scientific Objectives and Requirements}

The main scientific objectives and experiment requirements of the CFVib experiment are described here, including the parameters and constraints of the parabolic flight campaign.

\section{Primary Scientific Objectives}

As stated above, the principal objective of the CFVib experiment is to investigate the complex behaviour of vibrated liquids in microgravity and to evaluate the potential for small-amplitude vibrations in controlling this behaviour. More specifically, the main scientific objectives are:

(i) Help evaluate the feasibility of managing fluids using small-amplitude vibrations in microgravity. In this regard, two different frequency regimes are considered: acoustic (high frequency) and hydrodynamic (low frequency).

(ii) Allow a comparison between different representative fluid configurations having distinct surface energies and wetting characteristics, including water-air, oilair, and liquid-liquid. Comparison is also made with unforced samples to differentiate the effect of the applied vibrations from that of residual ambient accelerations (like g-jitter).

(iii) Provide experience in the design and execution of a microgravity experiment centered on the vibroequilibria effect. Strengths and weaknesses of the design can suggest improvements for future microgravity experiments.

\section{Scientific Requirements}

The main scientific requirements of the experiment are outlined below. These relate to fluid properties, time constraints of the parabolic flight, sensitivity to g-level cycles and residual accelerations, forcing requirements, and observation criteria.

\section{Experimental Fluids}

The experiment considers different liquid-gas and liquidliquid configurations, which, in a qualitative sense, are representative of common space applications. Configurations include the case of a wetting fluid like silicone oil and a non-wetting fluid like water. The experiment fluids satisfy the safety requirements of the parabolic flight environment and are contained in relatively small, closed, transparent acrylic containers.

\section{Relaxation Time}

There are two potentially distinct processes whose timescales must be smaller than the available microgravity time.

(i) The decay time needed to reach the initial "static" configuration at the beginning of the microgravity phase. This time depends primarily on the damping experienced by the excited sloshing modes.

(ii) The vibroequilibria timescale needed for the vibrated fluid to reach a new quasi-static equilibrium.

Calculations (Weislogel and Ross 1990) using a cylindrical container comparable to the size of those in the CFVib experiment predict that the unforced sloshing mode decay time is on the order of 1-4 s, depending on the fluid viscosity, contact angle, surface tension and cylinder radius. In a rectangular container, numerical simulations of the full Navier-Stokes equations, like that shown in Fig. 1, give similar timescales (on the order of $2 \mathrm{~s}$ for a fluid with a viscosity of $50 \mathrm{cSt}$ ).

Once the vibrational forcing begins, the time needed for the fluid to approach the vibroequilibria state is, according to numerical simulations like that of Fig. 1, similar to the unforced relaxation time (on the order of several seconds). Although the dynamics are somewhat different with 

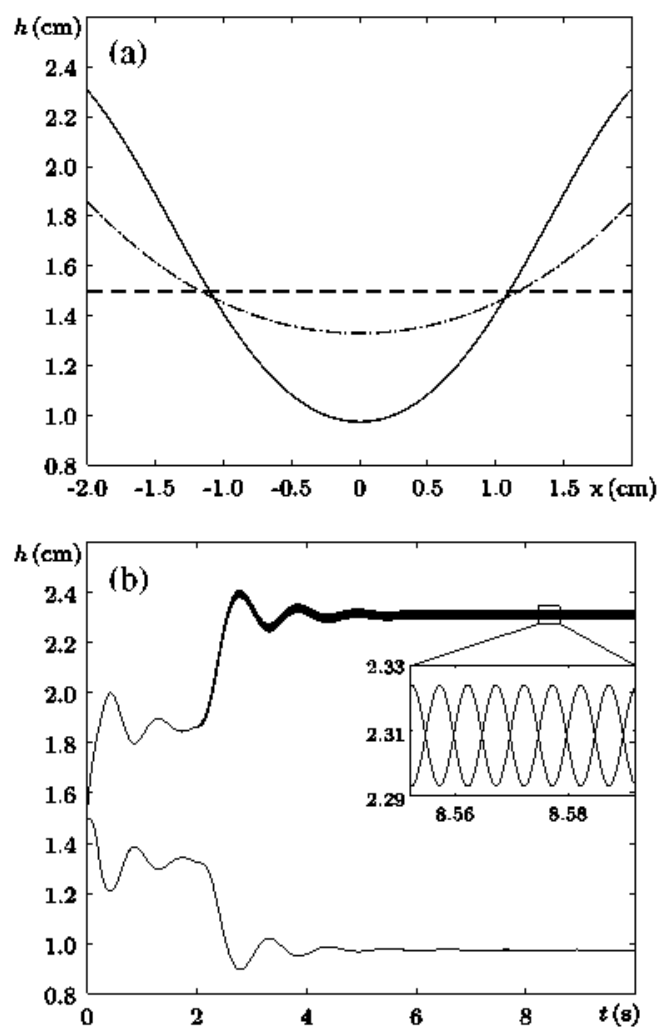

Fig. 1 Relaxation of a 2D fluid in a $4 \mathrm{~cm}$ wide container (fluid area/volume $6 \mathrm{~cm}^{2}$ ) subjected to horizontal vibrations at $100 \mathrm{~Hz}$ in the absence of gravity: forcing starts at $t=2 \mathrm{~s}$, viscosity is $50 \mathrm{cSt}$, surface tension 20 dyne $/ \mathrm{cm}$, and contact angle $60^{\circ}$. (a) Surface profile $h(x)$ at $t=0$ (initial flat surface: dashed line), at $t=2 \mathrm{~s}$ (hydrostatic surface: dash-dotted curve), and for large time (vibroequilibria surface; solid curve). (b) Heights $h(t)$ of the two contact points (upper curves) and midpoint (lower curve) showing relaxation to the hydrostatic solution $(t<2 s)$ and to the vibroequilibria solution $(t>2 s)$. The inset shows the out-of-phase harmonic waves at the two walls (one grey, one black)

the CFVib configuration using piezoelectric actuators, and depend as well on the resonance mode selected, it is likely that the streaming flow responsible for driving the fluid to its vibroequilibria state is established quite rapidly and that the relaxation process is limited by sloshing motion in both the forced and unforced cases. The sloshing frequency and effective damping clearly depend on the asymptotic state about which the oscillations occur, and thus on the vibrational forcing, but in most cases, this dependence is relatively weak.

Since the characteristic relaxation time is on the order of several seconds, both to settle into an initial unforced microgravity configuration and to evolve toward the vibroequilibria state when the forcing is turned on, this constitutes the basic time requirement of the CFVib experiment. Parabolic flights provide a period of microgravity of more than $20 \mathrm{~s}$ during each parabola, which is sufficient to observe the asymptotic behaviour.
Airplane Disturbances: g-level Cycles and g-jitter

The changing g-levels during a parabolic flight affect the position of the fluids in the experimental containers. Consequently, the configuration prior to each experimental run has a degree of unpredictability, as does the dynamics of the relaxation (sloshing) phase during the first few seconds of microgravity. One important scientific requirement is that g-level cycles and ambient g-jitter effects not mask or otherwise adversely affect the important experimental measurements.

Figure 2 shows a typical acceleration profile during the microgravity portion of a parabolic flight. The smallamplitude ambient accelerations that are present (amplitudes on the order of $0.05 \mathrm{~g}$ ) at low frequencies like $1 \mathrm{~Hz}$ are particularly worrying as a potential source of fluid motion and experimental errors since these frequencies are comparable to the relevant sloshing frequencies. Higher-frequency accelerations, known as "quickly changing acceleration forces" (QCAFs) (Schmidt 2004), are not captured in Fig. 2, but are not likely to adversely affect the scientific output of the experiment.

The g-level cycles, including high-g portions of the parabolic flight and small periods of negative $g$, are not detrimental to the success of the experiment since the initial conditions for the microgravity phase of the flight do not need to be known precisely. Although both g-level cycles and g-jitter may complicate precise quantitative comparison of different runs, this variation is compatible with the scientific objective of the CFVib experiment to investigate a general robust method for moving fluids in microgravity. The presence of unforced fluids in otherwise (nearly) identical experimental cells allows the importance of low-frequency noise and g-jitter to be directly evaluated.

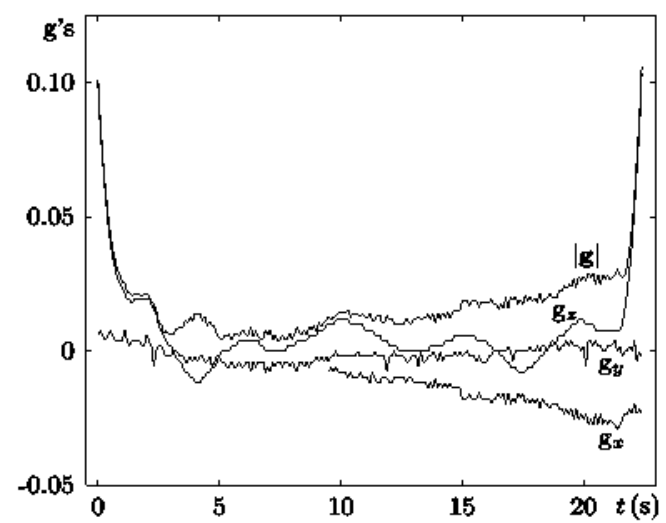

Fig. 2 Acceleration (in g's) during the microgravity portion of the parabolic flight in a 3-axis reference frame attached to the airplane showing the $z$-axis ("vertical" direction, $g_{z}$, dark grey), $y$-axis ("lateral" ditection. $g_{y}$. medium grey), $x$-axis ("longitudinal" direction, $\mathrm{g}_{x}$, light grey) and total magnitude (|g|. black) 


\section{Frequency Regimes}

The CFVib experiment uses high-frequency vibrations in the range of $50 \mathrm{~Hz}$ to $12 \mathrm{kHz}$. There are thus two distinct excitation regimes:

(i) The hydrodynamic regime where compressibility can be neglected.

(ii) The compressible regime where acoustic waves are essential in determining the velocity field.

The hydrodynamic regime pertains when the fluid is driven at frequencies up to several $\mathrm{kHz}$ while the acoustic regime is associated with higher frequencies (tens of $\mathrm{kHz}$ or more). The manner in which the vibrational energy is delivered to the fluid in these two regimes is different, and it is of interest to compare the vibroequilibria effect in each case.

\section{Fluid Velocity}

Sufficient fluid velocity is required to clearly observe vibroequilibria effects. This means that the vibrational energy associated with changes in the rapidly oscillating velocity field be comparable (or greater), at least locally, to the characteristic variation in surface and contact energy terms. An induced velocity of approximately $10 \mathrm{~cm} / \mathrm{s}$ or more is needed. Although fluid velocity is often measured using PIV techniques [see, e.g., Hasegawa et al. (2009), where streaming flow excited by an acoustic levitator was observed], this is beyond the scope of the CFVib experiment; PIV measurements may be included in future extensions.

\section{Interface Observation}

The experiment requires a series of images showing the deformation of the surface. Movement on the order of $1 \mathrm{~mm}$ can be detected by the camera. Recording two different views of each sample allows for more possibilities in postprocessing of the images and (potential) reconstruction of the fluid interface. An example of the type of interface expected (obtained from numerical simulation) is shown in Fig. 3.

\section{Design Requirements and Considerations}

\section{Low-power Forcing Device with a Wide Frequency Envelope}

Piezoelectric devices are used to induce a vibrational velocity field in the experiment fluids. To avoid having to operate the piezoelectric material at its own (high) resonance frequency, a bender beam configuration is used instead of attaching the piezoelectric ceramics directly to the external wall of the recipients. With this configuration (see subsequent

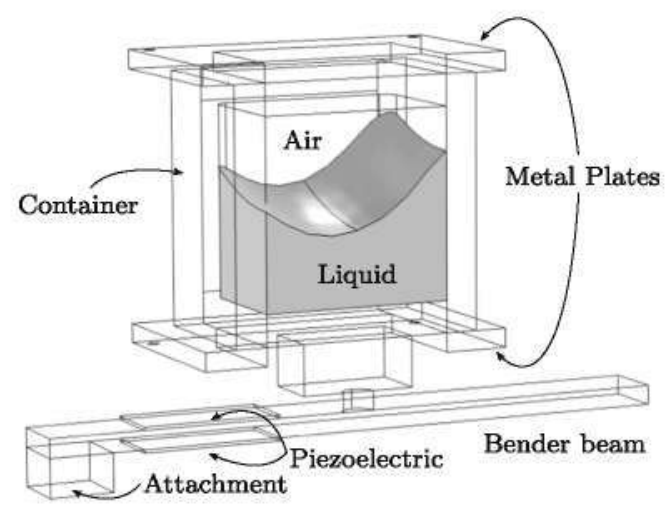

Fig. 3 Vibroequilibria solution with $88 \mathrm{~Hz}$ forcing (near the lowest resonance) and $60^{\circ}$ contact angle for a liquid with properties like water. The vibroequilibria surface is shown after $20 \mathrm{~s}$ while it takes about $4 \mathrm{~s}$, starting from the hydrostatic equilibrium, for transients to decay

sections for details), the resonant frequencies for prominent flexural (beam) and container modes range over several $\mathrm{Hz}$ to $\mathrm{kHz}$, as desired. Furthermore, forcing can be supplied by a cost-effective low-power amplifier.

\section{Interaction between Different Experimental Cells}

The effects of coupling between different experimental cells, which are mounted on the same base plate, are required to be no greater than those of the airplane disturbances ( $g$-jitter; see Fig. 2). Some degree of mechanical coupling between distinct experimental cells is unavoidable, and the importance of this effect, which is quite sensitive to resonances, is difficult to predict. Direct observations are made after the experiment is assembled.

\section{Real-time Data Acquisition and Visualization}

Since the excitation is (often) started after the capillary equilibrium is reached, it is an advantage for the experiment operator to monitor the fluid configuration directly in (nearly) real-time.

\section{Novespace Requirements}

Requirements like those summarized below help ensure safe experiment operation and compatibility with the airplane systems.

- Safety is strictly enforced during the parabolic flight and all potential hazards must be classified and analyzed.

- Electronic systems must ensure protection for operating personnel, aircraft interfaces, and the experiment itself. This is achieved through proper grounding, isolation of high voltage electrical connections, and the use of fuses in case of short circuits. 
- Liquids, powders and particles are required to be safe from accidental release into the airplane cabin; this contamination risk is reduced by a double containment policy.

- Structural hardware must withstand emergency-level forcing loads.

\section{Experiment Design}

The experimental set-up, sketched in Fig. 4 for a single experimental cell, includes the following main items:

(i) Four experiment fluids: distilled water, $5 \mathrm{cSt}$ silicone oil (Dow Corning), $50 \mathrm{cSt}$ silicone oil (Dow Corning), and vacuum oil (Krytox 1506).

(ii) Twelve experimental containers: six rectangular cuboids and six cylinders, half excited by vibration and half passive.

(iii) Twelve piezoelectric devices: two for each of the six excited containers, attached on opposite faces of the vibrating (bender) beam.

(iv) Four cameras, twelve mirrors and an LED light strip for video acquisition.

(v) Six accelerometers measuring the vibration of the excited cells.

(vi) Three (dual channel) wave generators, six acoustic amplifiers and an electronics board (including a DC Power Supply) driving the piezoelectric devices.

(vii) One laptop computer controlling the experiment.

\section{Experiment Fluids}

As mentioned above, one goal of the CFVib experiment is to evaluate the effect of surface tension and contact angle
Table 1 Characteristic physical properties of the experiment fluids at room temperature: density $(\rho)$, surface tension $(\Gamma)$, and kinematic viscosity $(\gamma)$

\begin{tabular}{llll}
\hline & $\rho\left(\mathrm{gm} / \mathrm{cm}^{3}\right)$ & $\Gamma($ dyne $/ \mathrm{cm})$ & $\gamma(\mathrm{cSt})$ \\
\hline Water & 1.0 & 72 & 1 \\
Silicone oil & 0.91 & 19.7 & 5 \\
Silicone oil & 0.96 & 20.8 & 50 \\
Krytox 1506 & 1.9 & 19 & 250 \\
\hline
\end{tabular}

in the selection of the vibroequilibria solution. In the CFE experiment (Jenson et al. 2010; Weislogel et al. 2009) the importance of the boundary condition in selecting the preferred hydrostatic configuration was shown. The CFVib experiment takes this into account, using different representative fluids with distinct surface and contact properties. The experiment uses water, two silicone oils of different viscosity, and a mixture of silicone oil and Krytox 1506 vacuum oil (which is about twice as dense as silicone oil). Characteristic physical properties of each experiment fluid are given in Table 1.

\section{Experiment Containers}

Two different experiment containers are used; a brief description of each is provided below.

\section{Cuboidal Containers}

Six rectangular cuboids made of methacrylate are used as containers for the experiment fluids. To protect against leaking, they are built using only two pieces: a body and a lid. The interface between the lid and the body is sealed using an

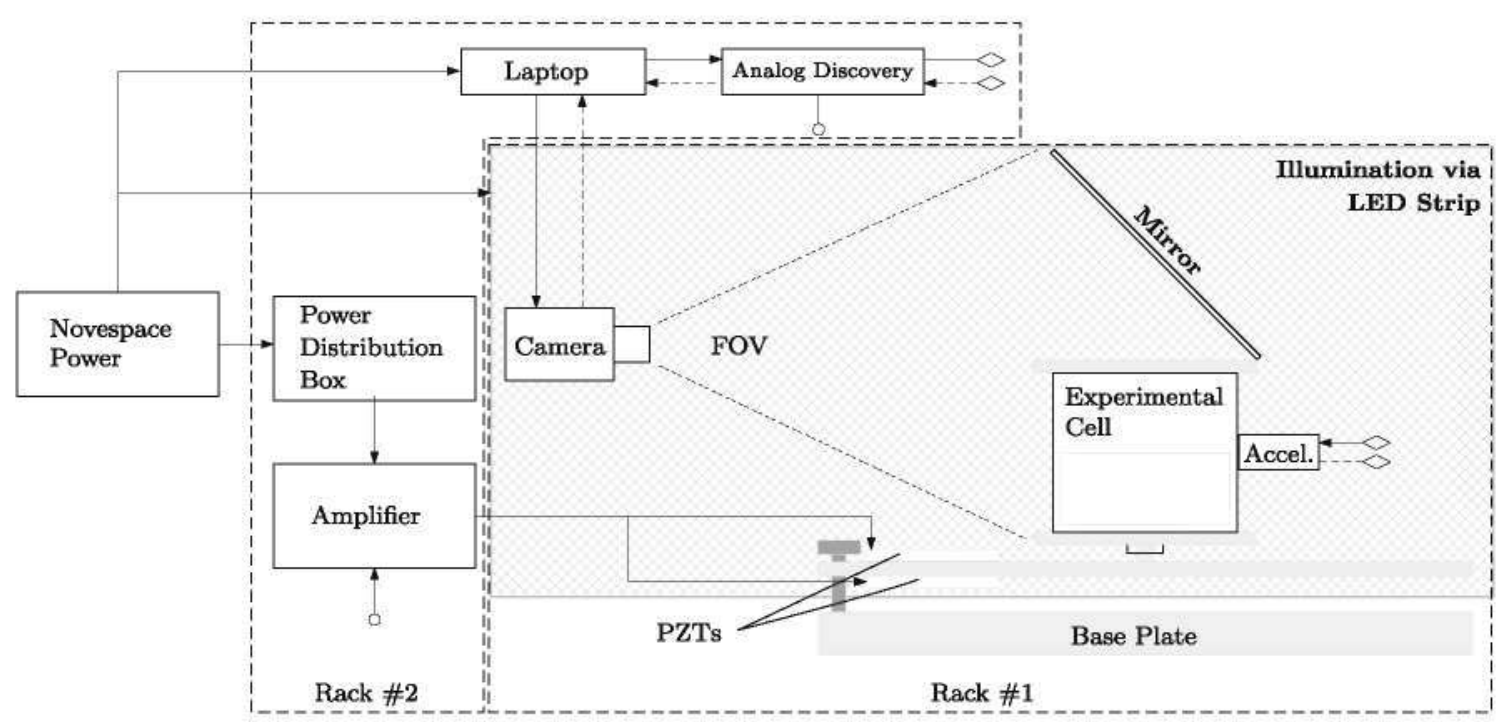

Fig. 4 Sketch of CFVib experiment showing just one cuboidal cell. Rack \#1 holds the experiment samples and main elements of the acquisition system. Rack \#2 holds the control and electronic components 
O-ring and pressure is maintained by two aluminum plates connected by four long bolts (see Fig. 5a). These containers have an interior dimension of $3 \times 3 \times 4 \mathrm{~cm}$ and a wall thickness of $0.5 \mathrm{~cm}$. They are approximately half-filled in the case of a single liquid, and full (no air) in case of the two-liquid mixture. The total volume of fluid is about $70 \mathrm{ml}$.

\section{Cylindrical Containers}

Six right circular cylinders made of $0.5 \mathrm{~cm}$ thick methacrylate are used as containers for the experimental fluids. The inner diameter is $3 \mathrm{~cm}$ and the height is $6 \mathrm{~cm}$. As with the cuboids, they are built using two pieces (body and lid) that are sealed with an O-ring and pressed together by two aluminum plates connected by long bolts (see Fig. 5b).

The six cylindrical containers have the same dimensions, as do the six regular cuboidal containers, respectively. Three of each type have a piezoelectric shaking device attached, while the other three are passive, providing an unforced reference configuration. The cuboidal cells are arranged at two different levels to optimize space on the base plate, while the cylindrical cells are at the same level. One container from each set of three has water, one (sometimes two) has silicone oil, and one (sometimes none) has a combination of silicone oil and Krytox vacuum oil.

\section{Piezoelectric Actuators}

Piezoelectric devices are used to induce the oscillatory velocity field in the experiment fluids. A bender configuration is selected for the reasons outlined in Table 2, including the need to shift the resonances from those of the piezoelectric ceramic itself.
Table 2 Comparison of relevant characteristics for alternative piezoelectric actuator set-ups

\begin{tabular}{lc}
\hline Direct attachment & Bender beam configuration \\
\hline - Expensive amplifier & - Acoustic amplifier \\
- Separate transducer & - Bender acts as transducer \\
- Thicker PZT ceramic & - Thinner PZT ceramic \\
(more expensive) & (less expensive) \\
- Simpler mechanical assembly & - More complex mechanical \\
& assembly \\
- Complex numerical analysis & - Complex numerical analysis \\
- No analytical solutions & - Some analytical solutions \\
- High resonance frequencies & - Some resonances in the range \\
& of interest \\
\hline
\end{tabular}

The shaking device used in the CFVib experiment is constructed from a pair of piezoelectric ceramic materials and a metal plate or strip, referred to as a bender, that is attached to one of the walls of the container. The ceramics are placed on opposite horizontal faces of the bender in order to induce flexural motion that (in the primary mode) moves the recipient up and down. This configuration requires antisymmetric excitation of the two piezoelectric devices (achieved via opposite polarization of the AC signal inputs). The longitudinal deformation created in the ceramics is transmitted to the beam as shear stress with opposite sign on opposing sides of the beam. This situation generates a linear shear stress distribution that, as predicted by Euler-Bernoulli theory in the limit of a slender (linear) beam, produces a purely flexural deformation (the integral related to longitudinal motion vanishes).

The bender, which is driven by the piezoelectric ceramics near one end, is attached to one of the walls of the
Fig. 5 Prototypes for (a) rectangular cuboidal recipient and (b) cylindrical recipient.
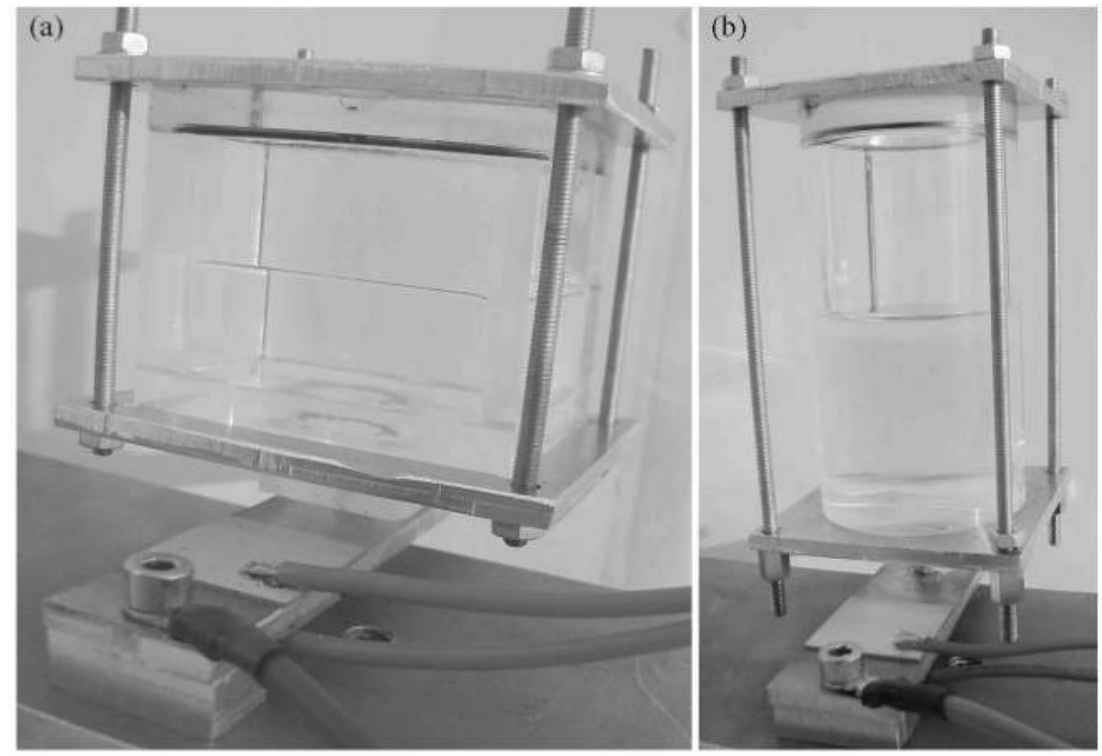
experimental container. This attachment is made by gluing a piece of methacrylate to the container and fixing this with a bolt to the cantilever plate. The bender is also clamped to the base plate with another bolted aluminum piece. Since in this set-up the individual experimental cells are not mechanically isolated and some degree of coupling between different bender-container assemblies occurs, a heavy base plate is used to reduce unwanted interaction.

\section{Data Acquisition: Cameras, Mirrors, Illumination, Accelerometers}

The data acquisition system includes the configuration of cameras, mirrors, illumination and accelerometers; each is described in more detail below. The cameras are powered via USB connection to the laptop. Videos showing the evolution of the surface are taken from two viewpoints for each cell. Accelerometers, controlled by Analog Discovery 2 devices (see "Electronics and Control Systems"), measure the effective vertical acceleration of the excited containers. Each set of three containers has a dedicated video camera (four video cameras in total) while illumination is provided by an LED strip.

\section{Video Cameras}

The video camera selected for the CFVib experiment is the ThorLabs DCC1545m, a CMOS camera that has a maximum frame rate of 25 FPS at the maximum resolution of $1280 \times 1024$ pixels. The MVL6WA camera lenses from ThorLabs are used with the camera. A high-speed camera is not required since the mean (averaged) position of the interfaces is observed, not the rapid dynamics occurring at the forcing frequency. A camera and lens pair with the same format $\left(1 / 2^{\prime \prime}\right)$ were selected to avoid cropping and vignetting of the images and to have the camera field of view (FOV) at the maximum allowed by the lens. The lens has a depth of field (DOF) of around $50 \mathrm{~mm}$, which allows the interior of the experimental cells to be observed. A summary of the main camera and lens characteristics is given in Table 3.

Table 3 Camera, lens and sensor characteristics

\begin{tabular}{lll}
\hline & Cuboids & Cylinders \\
\hline Format & $1 / 2^{\prime \prime}$ & $1 / 2^{\prime \prime}$ \\
FOV & 69.4 & 69.4 \\
Object distance $(\star)$ & $200 \mathrm{~mm}$ & $250 \mathrm{~mm}$ \\
Focal length & $6^{\prime \prime}$ & $6^{\prime \prime}$ \\
Horizontal Pixels/mm $(\star)$ & 9.24 & 7.39 \\
Vertical Pixels/mm $(\star)$ & 7.392 & 5.91 \\
\hline
\end{tabular}

( $\star$ Approximate numbers that vary somewhat in final configuration

\section{Illumination}

A commercial LED array is used for illumination. The model EGLO LED DECO is a $3 \mathrm{~m}$ LED strip which includes a $12 \mathrm{~V}$ power supply and has a maximum power consumption of $13.5 \mathrm{~W}$ with $250 \mathrm{~lm}$.

\section{Accelerometers}

Six Analog Devices ADXL001-500BEZ accelerometers are used to measure the vibration induced in the excited experimental cells. Each accelerometer is connected to one of the channels of an Analog Discovery 2 device, receiving the required $5 \mathrm{~V}$ and providing the measured signal. Two accelerometer measurements from the experiment are shown in Fig. 6.
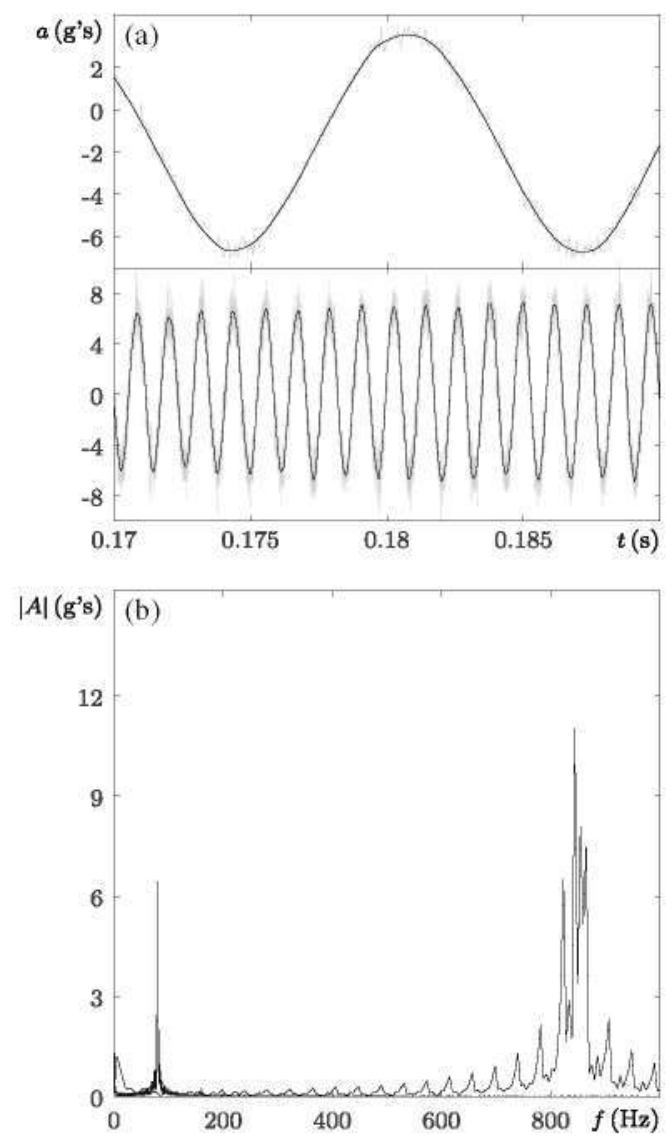

Fig. 6 Accelerometer measurements taken from the cuboidal cell with water during the third flight in parabolas 5 and 15 , using excitation frequencies of $79.8 \mathrm{~Hz}$ (first resonance) and $846.5 \mathrm{~Hz}$ (third resonance). (a) Time series of the accelerometer signals $a$ (in g's) for forcing at $79.8 \mathrm{~Hz}$ (upper panel) and $846.5 \mathrm{~Hz}$ (lower panel). Unfiltered (grey curves) and filtered signals (black curves) are shown. (b) Absolute value $|A|$ of the fast Fourier transform of both signals (lower frequency signal rendered with a thicker line) showing peaks at the corresponding excitation frequencies 


\section{Electronies and Control Systems}

The control and electrical systems, sketched in Fig. 4 and described in more detail below, include:

(i) Laptop computer

(ii) Amplifiers

(iii) Signal generators

(iv) Power supplies

(v) Other hardware: cabling, glands, etc.

Depending on the weight of the item, Velcro strips or mechanical straps are used to secure its position.

\section{Laptop}

A commercial high-performance MSI laptop computer is used to control the experiment and the piezoelectric actuators.

\section{High Voltage Amplifiers}

Six independent amplifiers are required. The amplifier selected is the PDm200B from PiezoDrive, which has the following features:

- Overload protection for current (at $0.5 \mathrm{~A}$ ) and temperature (at $70^{\circ} \mathrm{C}$ ).

- Maximum AC output voltage of $200 \mathrm{~V}$ and current of $300 \mathrm{~mA}$.

- Weight: $50 \mathrm{gm}$.

The amplifiers are placed in well-ventilated electrical boxes to allow effective cooling.

\section{Function Generator and Digital Oscilloscope}

Three Analog Discovery 2 devices are used to drive the piezoelectric ceramics. Because each one has two channels for arbitrary function generation and two oscilloscope channels for analog-to-digital conversion, it is used simultaneously to drive two piezoelectric actuators and to collect data from two accelerometers.

\section{Power Supply}

One TENMA 72-10505 DC Power Supply is used to provide the required voltage $( \pm 17 \mathrm{~V})$ to the amplifiers. In order to drive the six amplifiers, a power distribution box is used to obtain six lines.

The piezoelectric devices receive a sinusoidal electrical signal at the desired frequency produced by the Analog Discovery 2 function generators. The PDm200B amplifiers boost this to the required power level. Both systems are controlled and synchronized in time with the computer clock.
Operational Concept: Experimental Procedure, Software and Control

The experiment procedure is organized in accord with the sequence of separate microgravity periods presented by the parabolic flight plan. Each microgravity period is used to measure one experimental data point. The natural variables of the experiment include the container geometry (shape, size, aspect ratio), the fluid properties (viscosity, surface tension, density) and the excitation parameters (frequencies, amplitudes). The first two of these are constant between runs - different geometries (cuboids and cylinders) and different fluid configurations are considered simultaneously during the experiment.

The control variables of the experiment are the excitation parameters: frequency, $f$, and peak-to-peak voltage, $V_{\mathrm{pp}}$. The frequency selects the vibrational mode of the experimental assembly and, thus, the (qualitative shape of the) vibroequilibria solution reached, while, in the absence of instabilities or transitions, the voltage selects its amplitude. The movement of the interface is recorded with a video camera.

The basic procedure for each run is as follows:

1. Video acquisition is started several seconds before injection (i.e., several seconds prior to microgravity).

2. A wait of $5 \mathrm{~s}$ (maximum) may be implemented after the start of the microgravity phase while the static equilibrium is reached.

3. Forcing is applied (with fixed $f$ and $V_{\mathrm{pp}}$ ) and accelerometer measurements are recorded for at least $25 \mathrm{~s}$; a minimum of $15 \mathrm{~s}$ of data is obtained during the microgravity period. The behaviour of the fluids in hypergravity may also be observed.

4. The forcing and data acquisition (video and accelerometers) are stopped.

The applied vibration is different for different sets of runs in order to explore distinct modes of excitation occurring at different (resonance) frequencies. These resonances can be seen in Fig. 7 for the case of 50 cSt silicone oil in a cuboidal container. Since the resonance frequencies vary a bit depending on the assembly procedure (bolt tightness, e.g., being one variable) the forcing frequencies are determined on site (i.e., after the experiment is fully assembled). More than seven frequencies are explored over the first sixty parabolas. Roughly, these frequencies may be classified as follows:

(i) $50-100 \mathrm{~Hz}$ : near the first resonance.

(ii) $150-250 \mathrm{~Hz}$ : near the second resonance.

(iii) $500-1000 \mathrm{~Hz}$ : near the third resonance.

(iv) $2-5 \mathrm{kHz}$ : near the fourth resonance.

(v) $\sim 8 \mathrm{kHz}$ : contact line displacement (deformation) observed during ground testing.

(vi) $10-12 \mathrm{kHz}$ : strongly acoustic regime. 


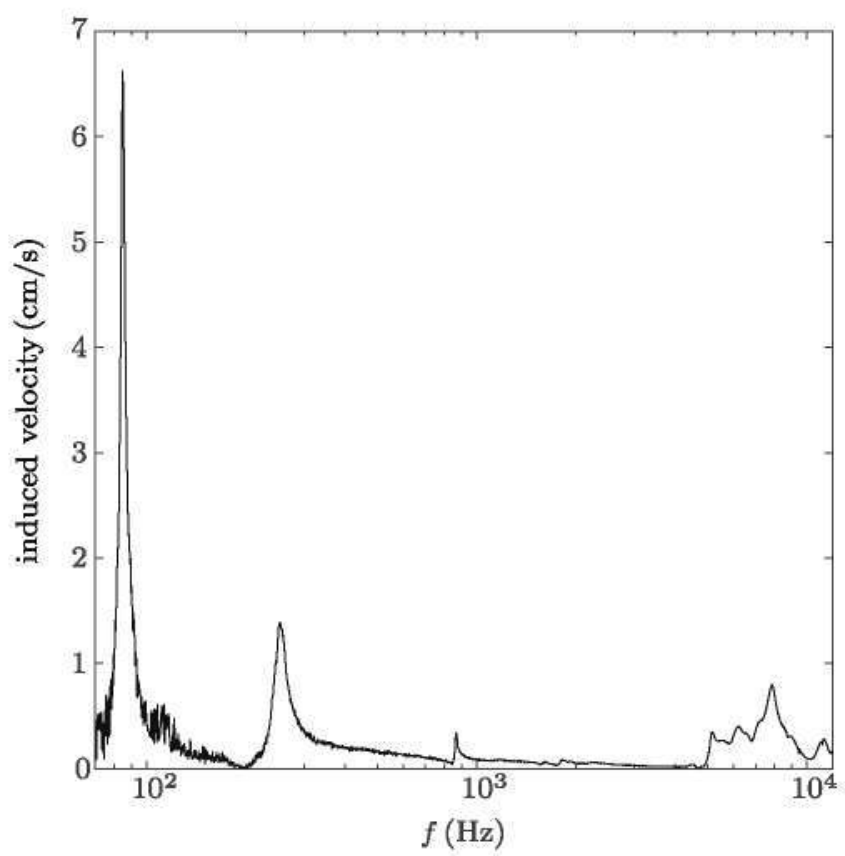

Fig. 7 Resonance behaviour in the cuboidal container with $50 \mathrm{cSt}$ silicone oil. Here $V_{p p}$ (applied to the piezoelectrics) is held at $40 \mathrm{~V}$ while the frequency $f$ is swept between $70 \mathrm{~Hz}$ and $12 \mathrm{kHz}$

Except for the first two resonances, where surface waves were seen during preliminary ground experiments and scientific interest is assured, the remaining frequencies are selected after the first flight ( 30 parabolas) - this is the only flight with a fully predefined experimental procedure. This flight is divided into two sets of ten runs and five sets of two runs. Over the two first sets of ten runs, the excitation amplitude (voltage) is increased linearly from an initially small value at a fixed frequency. These runs coincide with the first two resonances of the experimental assembly. For the remaining five sets of two runs each, various resonances over the range of interest are tested using intermediate $V_{\mathrm{pp}}$ values; depending on the results, they are selected (or not) for further study on subsequent flights. Acceleration values obtained during ground tests with different applied voltages for the first three resonance modes are shown in Fig. 8.

The final scientific data is contained in a compilation of four high quality videos (no compression) per parabola, six time series for the vertical acceleration (one per excited cell) and the accelerometer records of the airplane. This data is processed in a preliminary fashion between flights in order to decide on the selection of frequencies for subsequent flights. This processing includes:

(i) Analysis of the videos for these experimental runs, evaluating the degree of surface motion (compared with those of the corresponding unforced experimental cell).

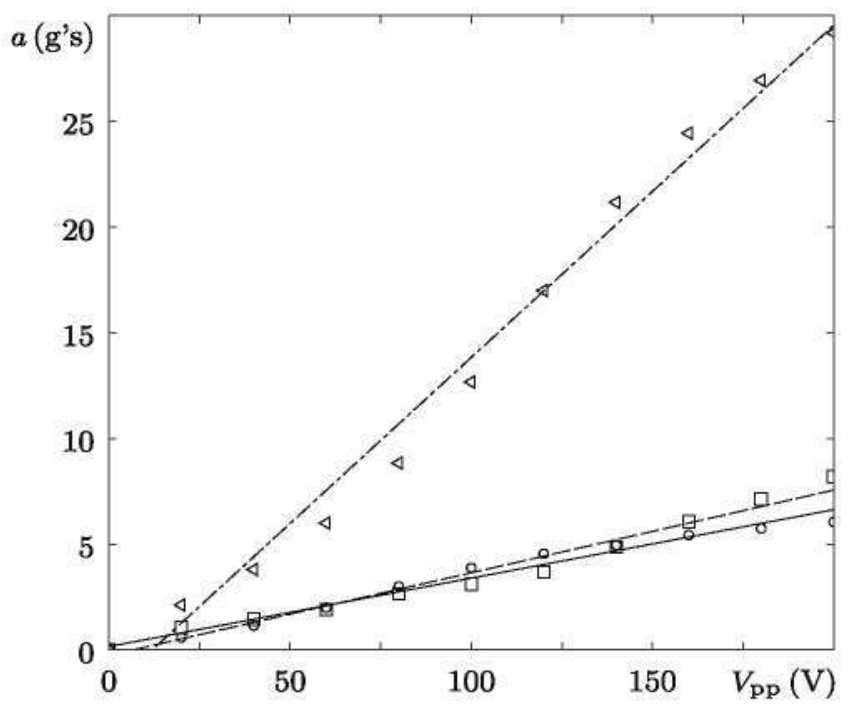

Fig. 8 Vertical acceleration $a$ (absolute value of harmonic component) measured during ground tests at the midpoint of the external rear wall for increasing (peak-to-peak) pieozoelectric excitation voltages $V_{\mathrm{pp}}$, at the first three resonance frequencies: $81 \mathrm{~Hz}$ (circles), $217 \mathrm{~Hz}$ (squares) and $841 \mathrm{~Hz}$ (triangles) of the cuboidal container with water. A linear fit is shown in each case

(ii) Analysis of the accelerometer data to estimate the fluid velocities induced.

Additionally, minor changes in the experimental procedure can be implemented by adjusting the vibration start/stop time, for example, or the set of runs (number of $V_{\mathrm{pp}}$ amplitudes measured for each frequency). Among other things, such possibilities are allowed for because the location of the resonances can shift due to the capillary driven relocation of the fluid and starting the vibration before injection (microgravity phase), when the interface is still relatively flat due to hypergravity, may help mitigate this effect.

\section{Review of Final Configuration}

The final configuration of the CFVib experiment follows from integrating the subsystems described above, as well as additional hardware and material related to safety requirements. The heart of the experiment is a set of six cuboidal and six cylindrical methacrylate cells. Half of the experimental cells are vibrated using a PZT piezoelectric material attached via an aluminum bender. The other half of the cells are for reference and are not forced; although there is no piezoelectric actuator, the rest of the mechanical assembly is (nearly) identical to that of the forced cells. Each specific fluid configuration used, such as water-air for example, is found in a total of four experimental cells: two forced 
cells of different shape (cuboidal and cylindrical) and two corresponding unforced cells.

Because each excited fluid cell may have (slightly) different resonance frequencies when fully assembled, each piezoelectric actuator is operated independently. Specially designed software allows for independent frequency control while switching all piezoelectric devices on (and off) simultaneously. The experimental containers (both excited and reference) are observed using four cameras (controlled by the software), twelve mirrors and an LED illumination system.

The experiment hardware described, as well as the cabling, USB hubs, and the structures supporting the mirrors, is placed inside a Zarges box and attached to a base plate in the airplane. This Zarges box, referred to as Rack\#1, contains the experimental cells, cameras, mirrors, and related hardware.

Rack\#1 is connected by a series of cables to the electronics and control assembly, placed on Rack \#2. Six power cables transmit the forcing signal, three Ethernet cables connect to the accelerometers (two for each cable), USB cables allow communication with the four cameras, and an additional power cable supplies the LED lighting. Each cable is passed through one of four waterproof glands and attached to the interior base plate to avoid inadvertent contact with the experimental hardware.

The base of Rack \#2 is a large aluminum plate where the different items of equipment are attached; this plate is directly joined to the airplane. Rack \#2 includes the Laptop, two electrical boxes containing the six amplifiers, a power distribution box, three Analog Discovery 2 devices, one Power Supply Unit, one USB Hub, the LED lighting remote control, and the electrical power strip.
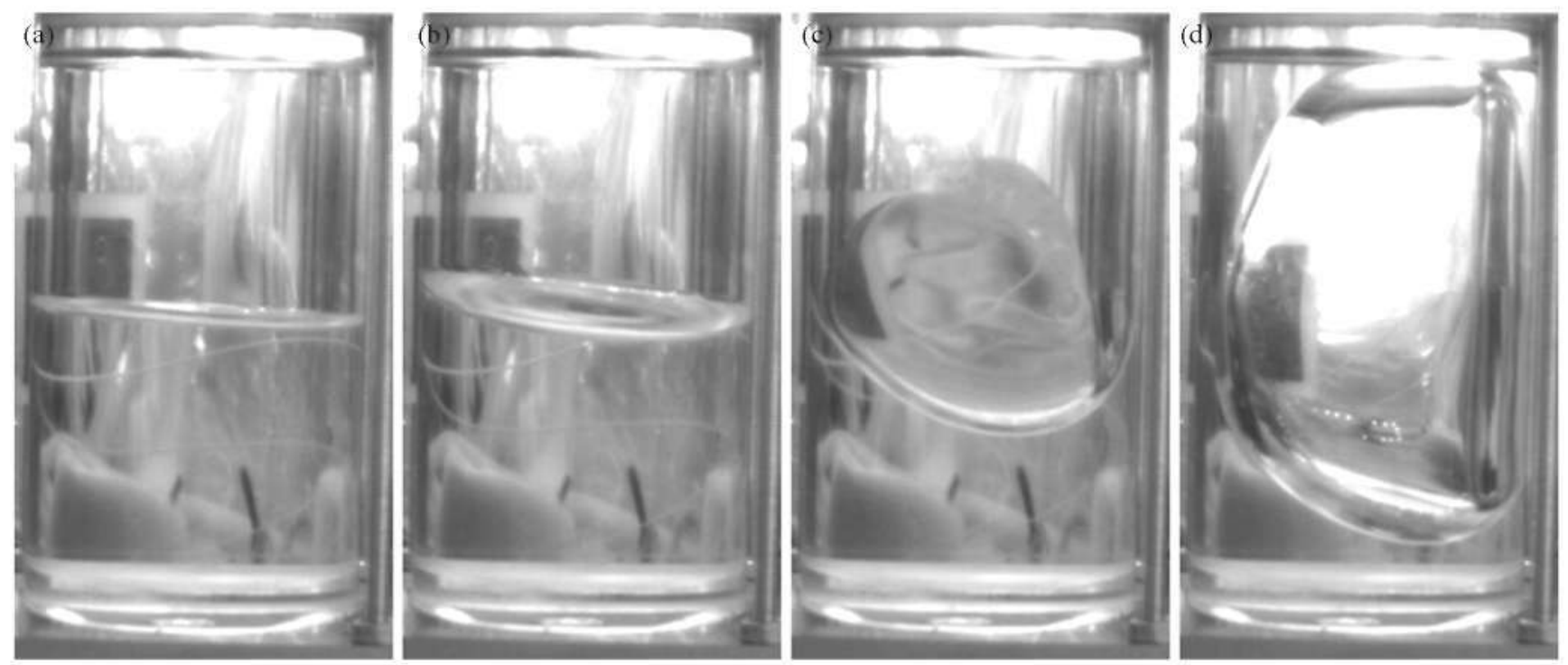

Fig. 9 Four snapshots from the experiment with $50 \mathrm{cSt}$ silicone oil vibrated at $59.3 \mathrm{~Hz}$ in a cylindrical container: (a) At the beginning of microgravity, (b) Just before forcing is applied, (c) About $2 \mathrm{~s}$ after forcing of amplitude $18.5 \mathrm{~cm} / \mathrm{s}$ is initiated, (d) About $4 \mathrm{~s}$ after forcing is initiated 
(v) The piezoelectric actuators were generally effective, allowing controlled excitation over a wide range of frequencies. A variety of modes can be selected (even more if additional bender contigurations are used) and, thus, a variety of vibrational motion and fluid response investigated. Signal generation and amplification worked well over this large range of frequencies.

\section{Conclusions}

In this paper we described the scientific objectives and design of the Control of Fluids in Microgravity with Vibrations experiment, which investigates the complicated behaviour of fluids subjected to vibrations in a weightless environment. The experiment focuses on the vibroequilibria phenomenon, whereby an inhomogeneous oscillatory velocity field causes the fluid to move and adjust its average shape. The fact that the selected vibroequilibria surfaces are increasingly flattened (perpendicular to the axis of vibration) by the applied periodic forcing means that this effect can be considered as a type of artificial gravity in some situations, and as a potential means to control and manipulate fluids in microgravity.

As discussed above, although there has been substantial theoretical investigation of vibrated fluids, including the vibroequilibria effect, there are relatively few microgravity experiments examining this phenomenon [those of Ganiev et al. (1977) being a notable exception]. It is of interest to test the predictions of vibroequilibria theory directly and to look at potential instabilities of these quasi-stationary states, as has recently been done (Fernández et al. 2017) using numerical simulations.

The experiment was proposed to serve as a first step in a more systematic investigation of vibroequilibria phenomena in microgravity. The experimental set-up is designed to record the behaviour of several different fluid configutrations in six cuboidal and six cylindrical cells, only half of which are deliberately excited by vibrations. The forcing is delivered through a cantilever beam driven by a pair of piezoelectric PZT ceramics subjected to a counter-phase electrical signal. Locating the resonances of this piezoelectric actuator (where the most energy can be transferred to the fluid) is a critical part of the preparation of the experiment. Changes in vibrational energy associated with different interface configurations must be comparable to those due to contact and surface tension terms. The piezoelectric actuator used in the experiment allows for a wide range of frequencies to be investigated and the corresponding modes and vibroequilibria solutions include both hydrodynamic and acoustic cases. Different behaviour such as flattening or cratering can occur depending on the orientation of the dominant vibrational motion with respect to the initial surface.
Overall, the experiment was successful, providing a lot of useful data to compare with the predictions of vibroequilibria theory. As a full analysis of the results is completed, more specific modifications and improvements of both theoretical (numerical) models and microgravity experiment design will be suggested.

Acknowledgements This work was supported by the ESA Education Office in connection with the Fly Your Thesis! progranme and the 65th ESA Parabolic Flight Campaign, and by the Ministerio de Economía y Competitividad under Projects No. ESP2013-45432$P$ and No. ESP2015-70458-P. We thank the European Low Gravity Research Association (ELGRA) for their valuable scientific support and Novespace for all their technical advice and contributions during the project. We thank Trenz Electronic for their Analog Discovery device, Analog Devices (ADI) for providing the accelerometers, and Krytox ${ }^{\mathrm{TM}}$ Performance Lubricants. a business of The Chemours Company, for supplying the vacuum oil. Finally, we thank the Spanish Uset Support and Operations Centre (E-USOC) and the Escuela Técnica Superior de Ingeniería Aeronáutica y del Espacio at the Universidad Politécnica de Madrid, which have supported this project from the beginning.

\section{References}

Arbell. H. Fineberg, J.: Pattern formation in two-frequency forced parametric waves. Phys. Rev. E 65, 036224 (2002)

Barnard. B.J.S., Pritchard, W.G.: Cross-waves. Part 2. Experiments. J. Fluid Mech. 55, 245-255 (1972)

Benjamin. T.B., Ursell, F.: The stability of a plane free surface of a liquid in vertical periodic motion. Proc. R. Soc. Lond. A 225, 505515 (1954)

Beyer. K., Gawriljuk. I. Günther. M., Lukovsky. I.. Timokha, A.: Compressible potential flows with free boundaries. Part I: Vibrocapillary equilibria. Z. Angew. Math. Mech. 81(4), 261-271 (2001)

Beyer, K., Günther, M., Timokha, A.: Variational and finite element analysis of vibroequilibria. Comput. Methods Appl. Math 4(3), $290-323$ (2004)

Beysens, D.: Vibrations in space as an attificial gravity? Europhys. News 37(3), 22-25 (2006)

Callens, N., Ventura-Traveset, J., de Lophem. T.L., Lopez de Echazarreta, C., Pletser, V., van Loon. J.J.W.A.: ESA parabolic flights. drop tower and centrifuge opportunities for university students. Microgravity Sci. Technol. 23(2), 181-189 (2011)

Callens. N., Ha, L.. Galeone, P.: Benetits of ESA gravity-related hands-on programmes for university students' careers. Microgravity Sci. Technol. 28(5), 519-527 (2016)

Edwards, W.S., Fauve, S.: Patterns and quasi-patterns in the Faraday experiment. J. Fluid Mech. 278. 123-148 (1994)

Faraday, M.: On a peculiar class of acoustical figures; and on certain forms assumed by groups of particles upon vibrating elastic surfaces. Philos. Trans. R. Soc. Lond. 121, 299-340 (1831)

Feng, Z.C.: Transition to traveling waves from standing waves in a rectangular container subjected to horizontal excitations. Phys. Rev. Lett. 79(3). 415-418 (1997)

Fernández, J., Tinao, I., Porter. J. Laverón-Simavilla. A.: Instabilities of vibroequilibria in rectangular containers. Phys. Fluids 29, 024108 (2017)

Funakoshi, M., Inoue, S.: Surface waves due to resonant horizontal oscillation. J. Fluid Mech. 192, 219-247 (1988)

Gandikota, G. Chatain, D., Amiroudine, S., Lyubimova. T. Beysens, D.: Frozen-wave instability in near-critical hydrogen subjected to 
horizontal vibration under various gravity fields. Phys. Rev. E 89 , 012309 (2014)

Ganiev، R.F., Lakiza, V.D.. Tsapenko. A.S.: Dynamic behavior of the free liquid surface subject to vibrations under conditions of neatzero gravity. Sov. Appl. Mech. 13(5)، 499-503 (1977). translated from from Prikladnaya Mekhanika

Gaponenko, Y.A., Shevtsova, V.: Shape of diffusive interface under periodic excitations at different gravity levels. Microgravity Sci. Technol. 28(4). 431-439 (2016)

Gaponenko, Y.A.. Torregrosa, M., Yasnou, V., Mialdun, A.. Shevtsova. V.: Dynamics of the interface between miscible liquids subjected to horizontal vibration. J. Fluid Mech. 784, 342-372 (2015)

Garrabos, Y., Beysens, D., Lecoutre, C., Dejoan. A., Polezhaev, V., Emelianov, V.: Thermoconvectional phenomena induced by vibrations in supercritical $\mathrm{SF}_{6}$ under weightlessness. Phys. Rev. E 75. $056317(2007)$

Gartett, C.J.R.: On Cross-waves. J. Fluid Mech. 41, 837-849 (1970)

Gavrilyuk, I., Lukovsky, I., Timokha, A.: Two-dimensional variational vibroequilibria and faraday's drops. Z. Angew. Math. Phys. 55, 1015-1033 (2004)

Gresho, P.M., Sani, R.L.: The effects of gravity modulation on the stability of a heated fluid layer. J. Fluid Mech. 40. 783-806 (1970)

Hasegawa, K.. Abe. Y.. Kaneko, A.. Yamamoto, Y.. Aoki. K.: Visualization measurement of streaming flows associated with a singleacoustic levitator. Microgravity Sci. Technol. 21(1), 9 (2009)

Jenson, R.M., Weislogel, M.M., Klatte, J., Dreyer, M.E.: Dynamic fluid interface experiments aboard the intemational space station: Model benchmarking dataset. J. Spacecr. Rocket. 47(4)، 670-679 (2010)

Jones, A.F.: The generation of cross-waves in a long deep channel by parametric resonance. J. Fluid Mech. 138, 53-74 (1984)

Kapitza, P.L.: Pendulum with a vibrating suspension. Usp. Fiz. Nauk. 44, 7-15 (1951)

Kudrolli. A., Pier. B., Gollub, J.P.: Superlattice patterns in surface waves. Phys. D 123, 99-111 (1998)

Lyubimov, D.V., Cherepanov, A.A.: Development of a steady relief at the interface of fluids in a vibrational field. Fluid Dyn. Res. 21(6), 849-854 (1986). translated from Izvestiya Akademii Nauk SSSR. Mekhanika Zhidkosti i Gaza

Lyubimov، D. V. Cherepanov، A.A., Lyubimova, T.P., Roux. B.t Interface orienting by vibration. C. R. Acad. Sci. Paris Ser. IIb 325, 391-396 (1997)

Lyubimov, D.V., Lyubimova, T.P., Cherepanov, A.A.: Dynamics of Interfaces in Vibrational Fields. Fizmatlit, Russian (2003)

Mialdun, A., Ryzhkov. I.I.. Melnikov. D.E., Shevtsova. V.: Experimental evidence of thermal vibtational convection in a nonuniformly heated fluid in a reduced gravity environment. Phys. Rev. Lett. 101, 084501 (2008)

Miles, J., Henderson, D.: Parametrically forced surface waves. Annu. Rev. Fluid Mech. 22, 143-165 (1990)

Miles, J.W.: Resonantly forced surface waves in a circular containet. J. Fluid Mech. 149. 15-31 (1984)

Perez-Gracia, J.M., Porter, J., Varas, F., Vega, J.M.: Subharmonic capillary-gravity waves in large containers subject to horizontal vibrations. J. Fluid Mech. 739, 196-228 (2014)

Pletser, V., Rouquette. S., Friedrich, U., Clervoy. J.F. Gharib, T., Gai. F., Mora, C.: The first eutopean parabolic flight campaign with the Airbus A310ZERO-g. Microgravity Sci. Technol. 28(6), 587-601 (2016)
Porter. J. Tinao. I. Laveron-Simavilla. A.. Lopez, C.A.: Pattern selection in a horizontally vibrated containet. Fluid Dyn. Res. 44, $065501(2012)$

Porter, J., Tinao, I., Laverón-Simavilla, A., Rodríguez, J.: Onset patterns in a simple model of localized parametric forcing. Phys. Rev. E 88, 042913 (2013)

Salgado Sanchez, P. Porter, J.. Tinao. I., laverón-simavilla, A.: Dynamics of weakly coupled parametrically forced oscillators. Phys. Rev. E 94, 022216 (2016)

Schmidt, W: Quickly changing acceleration forces (QCAFs) vibration analysis on the A300 ZERO-g. Microgravity Sci. Technol. 15(1). 42 (2004)

Shevtsova, V.: IVIDIL Experiment onboard the ISS. Adv. Space Res. $46(5), 672-679(2010)$

Shevtsova, V., Gaponenko, Y., Melnikov، D. Ryzhkov, I. . Mialdun. A.: Study of thermoconvective flows induced by vibrations in reduced gravity. Acta Astronaut. 66, 166-173 (2010a)

Shevtsova, V., Ryzhkov, I.I., Melnikov, D.E., Gaponenko, Y.A.. Mialdun, A.: Experimental and theoretical study of vibrationinduced thermal convection in low gravity. J. Fluid Mech. 648 . $53-82$ (2010b)

Shevtsova, V., Mialdun, A., Melnikov، D. Ryzhkov, I. Gaponenko, Y., Saghir, Z. Lyubimova. T., Legros, J.C.: The IVIDIL experiment onboard the ISS: Thermodiffusion in the presence of controlled vibrations. C.R. Mec. 339, 310-317 (2011)

Shevtsova, V., Gaponenko, Y.A., Sechenyh, V., Melnikov, D.E., Lyubimova, T. Mialdun. A.: Dynamics of a binary mixture subjected to a temperature gradient and oscillatory forcing. J. Fluid Mech. 767. 290-322 (2015)

Shevtsova, V., Gaponenko, Y.A., Yasnou, V., Mialdun, A.. Neponnyashchy, A.: Two-scale wave patterns on a periodically excited miscible liquid-liquid interface. J. Fluid Mech. 795. $409-422(2016)$

Talib. E.. Jalikop, S.V., Juel. A.: The influence of viscosity on the frozen wave instability: theory and experiment. J. Fluid Mech. $\mathbf{5 8 4}, 45-68$ (2007)

Tinao, I., Porter, J., Laverón-Simavilla, A., Fernández, J.: Cross-waves excited by distributed forcing in the gravity-capillary regime. Phys. Fluids 26.024111 (2014)

Varas. F., Vega, J.M.: Modulated surface waves in latge-aspect-ratio horizonally vibrated containers. J. Fluid. Mech. 579, 271-304 (2007)

Vega, J.M., Knobloch, E., Martel, C.: Nearly inviscid Faraday waves in annular containers of moderately large aspect ratio. Phys. D 154, $313-336(2001)$

Weislogel. M.M., Ross. H.D.: Surface settling in partially filled containers upon step reduction in gravity. Tech. rep (1990)

Weislogel, M.M., Jenson, R., Chen, Y., Collicott, S.H., Klatte, J., Dreyer, M.: The capillary flow experiments aboard the International Space Station: Status. Acta Astronaut. 65. 861-869 (2009)

Wolf. G.H.: The dynamic stabilization of the Rayleigh-Taylor instability and the corresponding dynamic equilibrium. Z. Physik. 227. 291-300 (1969)

Wolf, G.H.: Dynamic stabilization of the interchange instability of a liquid-gas interface. Phys. Rev. Lett. 24, 444-446 (1970)

Zyuzgin. A.V., Ivanov. A.I., Polezhaev, V.I., Putin, G.F., Soboleva, E.B.. et al.: Convective motions in neat-critical fluids under real zero-gravity conditions. Cosm. Res. 39(2), 175-186 (2001) 